\title{
Aspectos Relacionados Com a Otimização do Treinamento Aeróbio Para o Alto Rendimento
}

\author{
Related Aspects of Aerobic Training Optimization for \\ High Performance
}

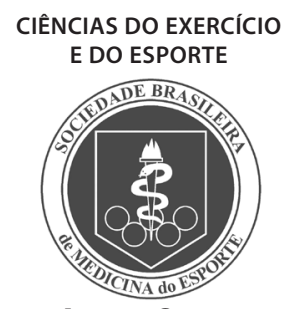

Artigo Original
Mariana Fernandes Mendes de

Oliveira'

Fabrizio Caputo

Camila Coelho Greco ${ }^{2}$

Benedito Sérgio Denadai²

1. Laboratório de Pesquisas em Desempenho Humano - Udesc, Florianópolis, SC.

2. Laboratório de Avaliação da Performance Humana - Unesp, Rio Claro, SP.

Endereço para correspondência: Fabrizio Caputo

Laboratório de Pesquisas em Desempenho Humano

Centro de Ciências da Saúde e do Esporte, Udesc.

Rua Pascoal Simone, 358

- Coqueiros - 88080-350 -

Florianópolis, SC.

E-mail: fabriziocaputo@hotmail.com

\begin{abstract}
RESUMO
O objetivo deste trabalho foi apresentar recomendações visando à otimização do treinamento aeróbio, a partir do conhecimento dos índices de aptidão funcional e seus mecanismos fisiológicos. Em atletas altamente treinados, a precisão na elaboração do treinamento pode ser o meio mais seguro para a melhora do rendimento, pois nesses indivíduos é comum a carga de treinamento oscilar entre o estimulo insuficiente e o aparecimento do excesso de treinamento. Existe, portanto, uma variedade muito grande de fatores que devem ser considerados na elaboração de um programa de treinamento. $O$ entendimento dos mecanismos de fadiga e das respostas fisiológicas associadas às diferentes durações e intensidades de exercício é essencial para uma correta elaboração das sessões de treinamento. Além disso, treinos intervalados de alta intensidade são imprescindíveis para melhora de rendimento em atletas altamente treinados, porém, é recomendado que ele seja realizado após um razoável período de recuperação das sessões de treino anteriores. Assim, o contato entre o atleta e o treinador é importante para um planejamento cuidadoso dos períodos de recuperação antes da ocorrência de fadiga excessiva. O treinador deveria arquivar um histórico das cargas de treino e recuperações, aprendendo com a própria experiência os tipos de cargas que podem ser toleradas individualmente. Entre os fatores que podem afetar o rendimento aeróbio, o planejamento de um aquecimento apropriado e as condições ambientais adversas são aspectos muito importantes. Após reunir todas essas informações, é possível elaborar as bases do treinamento (frequência, volume, intensidade e recuperação) visando melhora contínua do rendimento aeróbio.
\end{abstract}

Palavras-chave: fadiga, recuperação, treino intervalado de alta intensidade.

\begin{abstract}
The objective of this work was to present recommendations aiming the aerobic training optimization, from the knowledge of the indexes of functional fitness and their physiological mechanisms. Concerning highly trained athletes, the accuracy in training elaboration can be the safest way to improve aerobic performance, since for these individuals, it is normal that the training load is changeable between an insufficient stimulus and the overtraining syndrome symptoms onset. Therefore, there are several factors that should be taken into account for the elaboration of a training program. The knowledge on fatigue mechanisms and physiological responses at different exercise intensities and durations is essential for the correct training session elaboration. Moreover, high-intensity interval training is indispensable to improve performance in highly trained athletes; however, it should be performed only after adequate recovery period. Thus, a good relationship between coach and athlete is also important for planning suitable recovery periods prior to excessive fatigue. The coach should keep accurate records of training loads and recovery times, learning hence the kinds of loads that can be individually tolerated. Among the important factors that can affect aerobic performance during competition and should be considered, we can name appropriate warm-up planning and adverse environmental conditions. After collecting all this information, it is possible to elaborate the training bases (frequency, volume, intensity and recovery) aiming at progressive improvement of aerobic performance.
\end{abstract}

Keywords: fatigue, recovery, high-intensity interval training. 


\section{INTRODUÇÃO}

Em atletas altamente treinados, a precisão na elaboração da carga de treinamento pode ser o meio mais seguro para a melhora do rendimento. Nesses indivíduos é comum a carga de treinamento oscilar entre o estímulo insuficiente e o aparecimento do excesso de treinamento (overtraining). Dessa maneira, o entendimento dos mecanismos de fadiga e das respostas fisiológicas associadas às diferentes durações e intensidades é essencial para uma correta elaboração das sessões de treinamento. Além disso, alguns aspectos relacionados com a recuperação, sejam de um único estímulo agudo ou de uma a várias sessões de treino, são importantes para prescrição adequada do treinamento. Após reunir todas essas informações, é possível elaborar as bases do treinamento (frequência, volume, intensidade e recuperação) visando melhora contínua do rendimento aeróbio.

Muitos cientistas do esporte baseiam seus conselhos para atletas e técnicos levando em conta os princípios do treinamento desenvolvidos a partir de estudos realizados em indivíduos não treinados ou em atletas recreacionais ${ }^{(1)}$. Isso é problemático, já que mudanças rápidas no $\mathrm{VO}_{2}$ max, número de capilares sanguíneos e atividade enzimática comuns a esses indivíduos com menor nível de aptidão, não ocorrem quando atletas altamente treinados aumentam seu volume de treino submáximo ${ }^{(1)}$. Portanto, uma vez o indivíduo tenha alcançado valores de $\mathrm{VO}_{2}$ max acima de $60 \mathrm{ml} / \mathrm{kg} / \mathrm{min}$, o desempenho de resistência não é melhorado através de aumentos adicionais no volume de treino submáximo(2). Quanto maior for o estado de condicionamento, maiores serão as intensidades necessárias para promover e manter as adaptações. A utilização de altos volumes para que ocorram adaptações contínuas aumenta muito o risco de lesões e proporciona pouco desenvolvimento das fibras rápidas (tipo lla), que poderiam contribuir de maneira significativa para o rendimento aeróbio ${ }^{(3)}$. Em atletas muito bem treinados, é necessário incluir na semana algumas sessões de treinamento intervalado de alta intensidade ${ }^{(4)}$. Mas quanto e quando ele deve ser aplicado? O que fazer para otimizar esse treino intervalado de alta intensidade, que parece ser a única ferramenta capaz de causar adaptações em indivíduos altamente treinados? Portanto, o presente trabalho tem como objetivo apresentar recomendações visando a otimização e o controle do treinamento aeróbio, a partir do conhecimento dos índices de aptidão funcional e seus mecanismos fisiológicos.

\section{Otimizando o treinamento de alta intensidade}

Muito pouca informação temos disponível a respeito da otimização de um programa de treinamento intervalado de alta intensidade para indivíduos altamente treinados. Otimização refere-se à ótima intensidade de exercício, duração e número de esforços, como também à duração e ao tipo de recuperação (ativo vs. passivo) entre os esforços. Essas variáveis são manipuladas de acordo com a fase de periodização do treinamento, estado de treinamento e respostas individuais que o atleta apresenta.

Um grande número de variáveis tem sido estudado para prescrição da intensidade de exercício para indivíduos que realizam treinamento aeróbio. Entre as principais incluem-se o consumo máximo de oxigênio $\left(\mathrm{VO}_{2} \mathrm{max}\right)$, menor intensidade onde o $\mathrm{VO}_{2}$ max é atingido $\left(\mathrm{IVO}_{2} \mathrm{max}\right)^{(5)}$, máxima fase estável de lactato sanguíneo (MFEL) e limiar de lactato $(L L)^{(6,7)}$. Além dessas, uma opção muito atraente, de baixo custo, e que há muito tempo vem sendo utilizada empiricamente para prescrição da intensidade do treinamento, é a velocidade/intensidade que o atleta mantém durante a prova. Simplesmente colocar um atleta para se exercitar em intensidades próximas (na intensidade ou acima) àquela da prova, provavelmente, garantiria estímulos capazes de melhorar o rendimento na intensidade desejada. No entanto, essa maior "facilidade" de aplicar cargas de treinamento baseado na intensidade de prova não elimina a necessidade de conhecimento mais profundo das variáveis relacionadas com o treinamento. É muito importante que as pessoas envolvidas com a prescrição do treinamento saibam quais são as respostas fisiológicas referentes às intensidades de esforço, à contribuição relativa dos metabolismos energéticos envolvidos, ao "desgaste" metabólico/fisiológico e ao tempo de recuperação desse "desgaste", para que consigam chegar à programação mais efetiva de treinamento. Além disso, a simples utilização de índices fisiológicos, imaginando que esses seriam mais precisos do que outros métodos, não traria necessariamente o resultado esperado sem o correto entendimento dos mecanismos envolvidos.

Uma variável que vem sendo usada com sucesso em corredores e ciclistas para prescrição da intensidade do exercício é a $\mathrm{IVO}_{2} \mathrm{max}^{(8,9,10)}$. Uma das razões para usar a $\mathrm{IVO}_{2}$ max em um treinamento intervalado de alta intensidade (TIAI) é baseada no fato de que aumentos no $\mathrm{VO}_{2}$ max e, mais importante ainda, na performance de atletas altamente treinados, serão somente conseguidos treinando na $\mathrm{IVO}_{2}$ max ou acima dela. Além disso, o tempo máximo de exercício (Tlim) que um indivíduo consegue manter na $\mathrm{IVO}_{2}$ max tem sido utilizado para estabelecer a duração do esforço de maneira individualizada. TIAl realizados de uma a duas vezes na semana, com séries de quatro a seis tiros na $\mathrm{IVO}_{2}$ max, durações entre 50 e $60 \%$ do Tlim na $\mathrm{IVO}_{2}$ max, uma relação de tempo esforço:pausa de 1:2 ou 1:1, e a pausa sendo realizada de forma ativa a $50 \%$ da $\mathrm{IVO}_{2}$ max, parecem ser ótimos para melhora do rendimento aeróbio $^{(10)}$. Treinos ainda mais intensos, realizados entre 130 e 250\% da $\mathrm{IVO}_{2}$ max, e de curta duração (entre 15 e 60s), também têm efetivamente melhorado o desempenho aeróbio ${ }^{(3,5)}$. Portanto, a principal vantagem do treino intervalado seria a capacidade de poder realizar um número muito grande de combinações entre duração e intensidade do esforço vs. duração e intensidade da pausa, a fim de que o atleta possa se exercitar o maior tempo possível na intensidade requerida. Com isso, conseguiria obter maior estímulo para as adaptações necessárias à melhora do rendimento. Nesses treinamentos mais intensos, maior quantidade de fibras do tipo II (glicolíticas) é recrutada e elas acabam sendo induzidas a trabalhar de forma aeróbia. As fibras do tipo II são conhecidas por produzir mais lactato e, consequentemente, depletar os estoques de glicogênio mais rapidamente. No entanto, essas fibras têm velocidades muito mais altas de ressíntese de glicogênio, além de ser capazes de utilizar grande quantidade de lactato para reconversão ao glicogênio(11,12). Assim, o TIAl, além de estimular as fibras do tipo ॥ a trabalharem aerobiamente, aumentando a sua capacidade oxidativa (interconversão de fibras IIb para lla) e contribuindo consideravelmente para o aumento da capacidade oxidativa total, poderia ser também um excelente estímulo para acelerar a síntese de glicogênio.

Um aspecto importante é que esses treinamentos intervalados de alta intensidade devem ser realizados quando os atletas não estão com os estoques de glicogênio depletados, isto é, recuperados das sessões de treinos anteriores e capazes de realizar o treinamento na intensidade desejada. Observando a utilização do glicogênio muscular para uma correta organização desses treinos intensos durante a semana, foi demonstrado que 10 tiros de 1 min, realizados a $150 \%$ do VO $\mathrm{V}_{2}$ ax, com 2 min de pausa conseguiram praticamente depletar os estoques de glicogênio muscular ${ }^{(13)}$. Em outro exemplo, apenas três repetições máximas de 30s ( 250\% do VO $\mathrm{V}_{2}$ max) foram capazes de reduzir o glicogênio muscular pela metade ${ }^{(14)}$. Quando o glicogênio muscular atinge valores reduzidos, ocorre diminuição da intensidade do exercício, devido inicialmente à necessidade de deslocamento do sistema anaeróbio glicolítico para o aeróbio. Posteriormente, dependendo da duração e intensidade do exercício (nível de depleção de glicogênio), um percentual maior do substrato energético utilizado é deslocado para as gorduras, que possuem velocidade mais baixa de ressíntese de $\operatorname{ATP}^{(15,16)}$. 
Com mais sessões de treinos desafiadoras, a tolerância passa a ser específica para cada indivíduo. Assim, com o tempo, os efeitos das diferenças na genética, aptidão inicial e velocidade de adaptação causarão uma taxa variável de melhora. Essa variabilidade será mais evidente com as sessões mais difíceis. A sensibilidade em perceber essa variabilidade prevenirá atletas, que respondem mais lentamente às adaptações, de lesões, excesso de treinamento ou de se tornarem frustrados. Sessões mais individualizadas, principalmente nos últimos estágios de um planejamento, podem ser a melhor maneira de aperfeiçoar determinada aptidão. O melhor método para identificar esses detalhes específicos da resposta ao treinamento e melhorar o planejamento (rendimento final) é manter um detalhado diário de treino. As conclusões de um macrociclo (temporada) podem e devem ser aplicadas para melhorar estratégias para a próxima temporada.

\section{Período de recuperação}

O conhecimento do quanto cada sistema energético é utilizado em determinado treino, da quantidade de metabólitos acumulados e substratos energéticos utilizados, e o tempo que leva para recuperar cada uma dessas variáveis são aspectos importantes no treinamento. Infelizmente, muito pouca informação está disponível em relação ao período ótimo de recuperação entre os estímulos de um TIAl. Geralmente, técnicos e pesquisadores têm usado uma relação de tempo esforço pausa fixa, por exemplo 3:1, 2:1 1:1, 1:2, ou tempo de recuperação baseado no retorno da frequência cardíaca a determinada porcentagem da FCmax (geralmente 60\%) ${ }^{(9)}$. A escolha dessa relação deve ser feita em função do objetivo de cada sessão de treinamento, lembrando que, dependendo da intensidade, os períodos incompletos de recuperação fazem com que ocorra uma mudança de predomínio do metabolismo energético à medida que determinado sistema energético vai fatigando ou que a duração do exercício aumenta ${ }^{(17,18)}$.

A consequência disso é que essas mudanças de predomínio dos sistemas energéticos obrigam o indivíduo a diminuir a intensidade do exercício realizado de forma máxima. Como exemplo, isso acontece quando o atleta realiza séries de exercícios nos quais precisa sempre percorrer determinada distância da maneira mais rápida possível, mesmo que a velocidade diminua de uma repetição para a outra. A vantagem de utilizar períodos incompletos de recuperação é que durações relativamente curtas de exercício (com característica inicial anaeróbia) podem ser realizadas em maior intensidade e com o predomínio do metabolismo aeróbio ${ }^{(19)}$. Geralmente, a recuperação entre os intervalos deve ser longa o suficiente para permitir que o rendimento da próxima repetição continue a ser realizada na velocidade desejada. Para esforços mais longos realizados em velocidades mais lentas, a recuperação será mais curta que a duração do esforço. Para esforços bem curtos e intensos, períodos mais longos que o próprio esforço podem ser apropriados.

Recentemente, Gibala et al. ${ }^{(20)}$ utilizaram um modelo de treinamento que consistia em quatro a seis repetições de 30s máximos ( $250 \% \mathrm{VO}_{2}$ max) com quatro minutos de recuperação. Apesar de o intervalo de recuperação não ter sido suficiente para manutenção da potência média entre as repetições, esse tipo de treinamento diminuiu em 10\% o tempo para realização de um contrarrelógio de $750 \mathrm{~kJ}$ ( $60 \mathrm{~min}$ ), com apenas seis sessões de treinamento (duas semanas), em indivíduos ativos. Portanto, dependendo do tipo de treinamento, a manutenção da intensidade durante todas as repetições pode não ser o fator crucial para causar as adaptações desejadas, o que no caso do estudo citado acima seria induzir grandes adaptações aeróbias ao invés de anaeróbias. Além disso, nesse mesmo estudo os autores demonstraram que as adaptações musculares (e.g., conteúdo de glicogênio e enzimas aeróbias) relacionadas com a melhora da capacidade de exercício foram semelhantes entre o treino citado anteriormente (intervalado de alta intensidade) e o treino tradicional contínuo de longa duração (90-120 min a 65\% $\mathrm{VO}_{2}$ max).
Devido à elevada produção de lactato durante os treinos intervalados acima da MFEL, a realização de uma recuperação ativa entre os esforços aumentará a remoção do lactato, permitindo aos atletas tolerar intensidades altas de esforço por períodos mais prolongados. Realmente, a tolerância ao desconforto causado pela acidose só pode ser desenvolvida através da experiência com ela. Em adição aos outros benefícios (aumento da densidade mitocondrial e no número de capilares sanguíneos), o treinamento intervalado estimula a taxa de remoção do lactato, o qual depende diretamente do seu nível e da quantidade de seus transportadores na membrana celular ${ }^{(12,21)}$. Nesse sentido, é importante destacar que os benefícios desses tipos de treinamento sobre a capacidade e potência aeróbia não são dependentes somente do tempo gasto no $\mathrm{VO}_{2}$ max, geralmente o critério escolhido para elaboração das sessões de treinamento, mas também do tempo mantido em alta intensidade independente do valor de $\mathrm{VO}_{2}$ atingido.

Após um treino de alta intensidade, apenas com o repouso seria possível retornar os valores de lactato ao seu nível basal. O lactato sanguíneo tem uma meia-vida em torno de 15 min durante a recuperação passiva (repouso) ${ }^{(21)}$; dessa forma, após um exercício muito intenso os valores de repouso serão atingidos em aproximadamente 90 min. Assim qualquer tipo de recuperação realizada com intuito de aumentar a remoção do lactato entre sessões de treinamento parece supérfluo. No entanto, durante competições onde o atleta realiza mais de uma prova, a utilização de um exercício muito leve é aconselhada para auxiliar na remoção de metabólitos (figura 1), sem utilizar quantidades significativas de glicogênio.

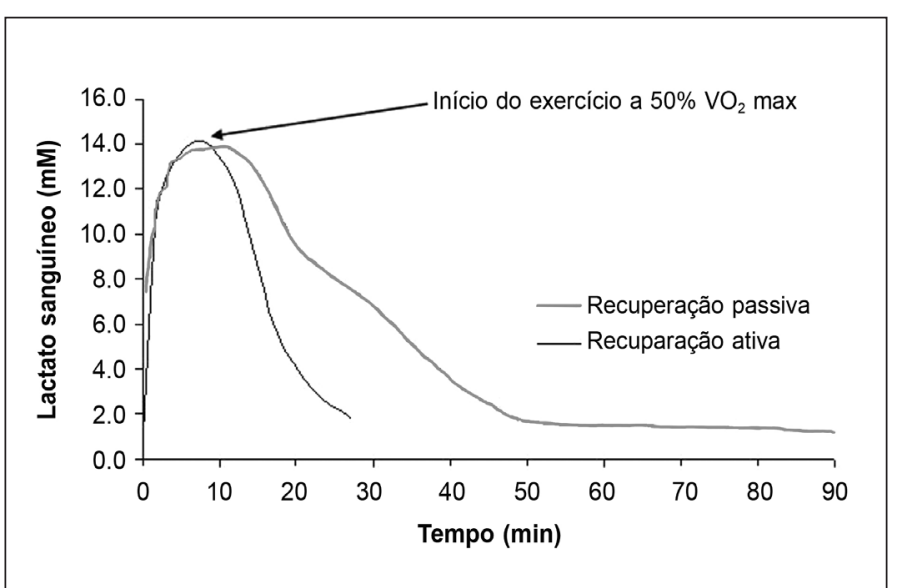

Figura 1. Efeito do tipo de recuperação sobre a remoção do lactato sanguíneo após um exercício máximo de $1 \mathrm{~min}$. A recuperação ativa foi realizada a uma intensidade correspondente a $50 \%$ do $\mathrm{VO}_{2}$ max e se iniciou 7 min após o término do exercício máximo. Dados não publicados coletados em nosso laboratório. É importante ressaltar que a recuperação ativa se iniciou no 7ํ min para propósitos de pesquisa e que a remoção do lactato seria mais rápida se a recuperação ativa iniciasse o mais rápido possível após o término do exercício máximo.

\section{Programação do treinamento}

Para a programação de um treinamento a frequência de estímulos (treinos) é um aspecto muito importante ${ }^{(22)}$. Se a frequência for muito baixa, o próximo estímulo será realizado após a fase de supercompensação, na qual já estaria ocorrendo uma queda da capacidade adquirida. Com esse tipo de periodização não seria esperado obter melhoras com o treinamento. Se a frequência for alta, os estímulos serão aplicados na fase de recuperação (antes do período de supercompensação), o que pode causar lesões, queda no condicionamento e, dependendo do caso, levar ao excesso de treinamento (overtraining) ${ }^{(23,24,25)}$.

Quanto maior é o estado de condicionamento de um atleta, mais frequentes devem ser os estímulos, principalmente devido ao aumento 
da velocidade de recuperação(26,27). Em atletas é muito comum ocorrer a soma de estímulos. Através de sessões de treinos consecutivos o estímulo pode ser maior e após um adequado período de recuperação poderá haver maior supercompensação(26) (figura 2, linha 2). Esse método tem sido usado, por exemplo, para induzir atletas a aumentar os estoques de glicogênio e a utilização de gorduras como substrato, através da sucessão de sessões de treinos intensos que conseguem diminuir os níveis de glicogênio a valores $\operatorname{críticos}^{(26,27)}$. No entanto, esse tipo de treinamento que provavelmente induza maiores adaptações deve ser realizado com muita cautela, para que não ocorram exageros nas intensidades e no número de treinos somados (figura 2, linha 3).

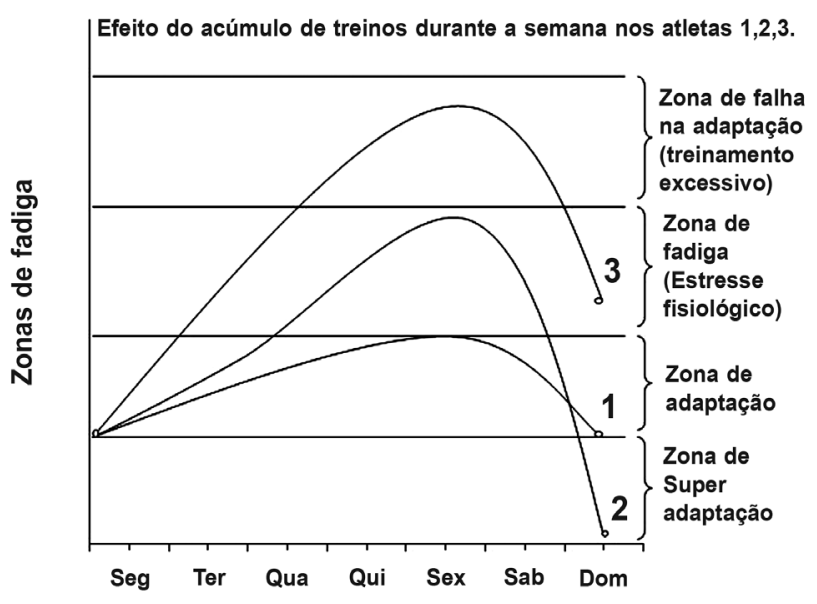

Figura 2. As três linhas (1, 2 e 3 ) indicam alterações no nível de fadiga em três diferentes atletas que treinaram por cinco dias consecutivos e descansaram ou fizeram um treino recuperativo no sábado e domingo (Adaptado de Counsilman (22))

Períodos adequados de recuperação, geralmente feita de forma ativa através de treinos leves, também devem ser utilizados e, dependendo do nível de desgaste atingido, podem durar mais que uma semana ${ }^{(28)}$. Além disso, com esse tipo de treinamento muitos componentes do sistema imune podem também exibir alterações adversas. Durante uma "janela aberta" de disfunção imune, que pode durar de três a 72 horas, dependendo do tipo de treinamento, vírus e bactérias podem ganhar espaço, aumentando o risco de possíveis infecções ${ }^{(29)}$. É muito comum nesse período atletas apresentarem infecções/infamações, principalmente das vias aéreas superiores ${ }^{(29)}$. Portanto, a ingestão adequada de nutrientes (antes, durante e logo após os treinos) e um período de sono adequado são imprescindíveis durante esse período de treinos muito intensos.

Um interessante estudo foi conduzido por Billat et al. ${ }^{(24)}$ avaliando corredores bem treinados que desenvolveram sinais indicativos de excesso de treinamento. Nesse trabalho os corredores treinaram durante um período de quatro semanas, realizando três sessões de treinamento intenso por semana (no $\mathrm{VO}_{2} \mathrm{max}$ ), além de um treino no limiar anaeróbio e dois treinos leves. O mais interessante foi que a melhora de rendimento proporcionada por apenas uma sessão de treinamento intenso por semana foi semelhante àquela demonstrada por três sessões. Nesse sentido, recomenda-se uma ou duas sessões por semana de treinamento de alta intensidade com no mínimo 36 horas entre as sessões. A razão para esse intervalo entre os treinos intensos se dá pelo fato de que, quando bastante depletado, o glicogênio muscular pode demorar de 12 a 36 horas para se recuperar ${ }^{(30)}$. Além disso, pequenos reparos estruturais nas mitocôndrias, fibras musculares, tendões e ligamentos, ocasionados principalmente pelas contrações excêntricas, podem levar de um a quatro dias para ser concluídos integralmente ${ }^{(28,31)}$, além de atrasar substancialmente a ressíntese de glicogênio ${ }^{(30)}$. No entanto, em casos mais extremos como provas de maratona, ultramaratona ou Ironman, foram encontrados indícios de microlesões mesmo 30 dias após a competição, justificando o grande período de recuperação que deveria ser dado após provas como essas ${ }^{(28)}$. Portanto, o volume do treinamento de alta intensidade deve ser aumentado gradativamente para permitir adaptação do sistema musculoesquelético. Ao mesmo tempo, devemse monitorar os sinais de respostas adversas ao treinamento, como, por exemplo, dores musculares após a sessão de treino. Quando qualquer atleta sente cansaço/dores nos músculos e articulações, sabe-se que um tempo maior de recuperação será necessário para refazer os estoques energéticos e a integridade dos tecidos conjuntivos.

Durante os dias nos quais o atleta apresenta dor muscular, a meIhor maneira de acelerar a recuperação parece ser o exercício muito leve ou repouso. A utilização de outras formas de recuperação, como massagens, não parece ser efetiva. Apesar de, para muitas pessoas subjetivamente, a massagem parecer auxiliar no relaxamento ou na recuperação, dados recentes demonstram que ela não é efetiva em aumentar o fluxo sanguíneo da musculatura, em acelerar a remoção do lactato, nem melhorar o rendimento. Ao contrário, a massagem é capaz apenas de aumentar o fluxo sanguíneo da pele, o que poderia deslocar parte do sangue da musculatura para a periferia(32).

A essência de aplicar com sucesso cargas apropriadas de treinamento é perceber antecipadamente quando será excessivo e então providenciar repouso periódico para assegurar que o treinamento sirva como um estímulo temporário ao invés de uma sobrecarga contínua. O repouso deve ser longo o suficiente para permitir a recuperação. Infelizmente, na maioria dos casos percebemos tardiamente que o estímulo foi excessivo, através de consequências patológicas (lesões ou infecções). Assim, manter um adequado diário das cargas de treino e períodos de recuperação, bem como notas relacionadas com a carga aplicada, é essencial. A síndrome do excesso de treinamento é causada por uma interrupção da habilidade do corpo em responder aos estresses "normais", tais como infecção ou treinamento ${ }^{(24,25)}$. É possível que essa grande anormalidade represente a resposta protetora de um corpo exaurido. Ao invés de sofrer danos adicionais, que seriam o resultado caso fosse permitido continuar o treinamento nesse estado depletado, o corpo responde de maneira a tornar o treinamento impossível. Portanto, para evitar que o treinamento seja prejudicial ao atleta, devemos estar mais atentos e aprender a respeitar as mensagens que o corpo nos oferece em resposta a um excesso de trabalho.

\section{Polimento}

Em atletas, a importância de um período de polimento seguindo uma fase de volume e intensidade de treinamento aumentado parece ser essencial. As reduções no volume, intensidade, frequência, que ocorrem durante o polimento podem afetar seriamente o desempenho. Quando a carga de treinamento é reduzida, a velocidade é perdida mais rapidamente que a resistência; assim, durante o polimento, reduzir o volume e manter ou aumentar a intensidade permitem recuperação completa e preparam o atleta para atingir o melhor desempenho durante a competição(33). A redução de $85 \%$ no volume de treinamento mostrou melhora na economia de corrida e no tempo da prova de $5.000 \mathrm{~m}$. A chave para um bom polimento é manter ou aumentar a intensidade do treinamento (> 85\% FCmax) enquanto reduz o volume no mínimo em 50\% ${ }^{(34)}$.

\section{Treinamentos em altitude}

Treinamento em altitude é frequentemente utilizado por técnicos e atletas na tentativa de melhorar o desempenho ao nível do mar. Para que ocorra melhora no desempenho é preciso que alguns efeitos da aclimatação em altitude ocorram: 1) aumento na oferta de oxigênio para o trabaIho muscular (primeiramente com aumento das células vermelhas); e/ou 2) melhora na utilização do oxigênio através da condição de hipóxia. 
Como visto anteriormente, os treinos intervalados de alta intensidade são importantes para melhora do rendimento, porém, muitas vezes os atletas não conseguem manter a intensidade do treinamento nessa altitude. Dessa forma, a exposição do atleta a um longo tempo de treinamento em altitude pode acarretar um destreinamento devido a redução do fluxo de oxigênio do capilar para a mitocôndria, podendo reduzir o estimulo adaptativo para a melhora da capacidade do músculo esquelético em extrair oxigênio. Pensando nesses aspectos, em meados de 1990, um novo modelo de treinamento para melhora do desempenho foi proposto; ele consistia em morar na altitude e treinar em baixa altitude.

Em uma série de estudos, atletas que moraram a uma altitude de $2.500 \mathrm{~m}$ durante quatro semanas demonstraram aumento de aproximadamente $7-8 \%$ das células vermelhas e aumento de $6 \%$ no VO $\mathrm{V}_{2}$ max em relação ao nível do mar. Contudo, apenas os atletas que treinaram em baixa altitude $(1.250 \mathrm{~m})$ demonstraram também manutenção do fluxo de oxigênio durante treinamento de base e treinamento intervalado, aumento de $7 \%$ do $\mathrm{VO}_{2}$ no limiar ventilatório e melhora na performance de corrida nos $3.000 \mathrm{~m}(1,5 \%)$ ou $5.000 \mathrm{~m}(3 \%)^{(35,36)}$.

Morar a uma altitude entre $2.000 \mathrm{~m}$ e $2.500 \mathrm{~m}$ parece ser melhor para maximizar o aumento dos glóbulos vermelhos no sangue enquanto minimiza as respostas complementares à aclimatação, as quais podem piorar o desempenho. Um mínimo de 12-16 horas de exposição à altitude parece ser necessário; contudo, quanto maior o tempo de exposição diária, melhor. Enquanto uma exposição de três semanas à altitude demonstrou significativo aumento das células vermelhas, uma exposição de quatro semanas demonstrou quase o dobro comparada com três semanas. Portanto, um mínimo de quatro semanas de exposição à altitude é recomendado(37).

Treinamentos de alta intensidade, como o TIAl ou contínuo em intensidades superiores ao $\mathrm{LL}$, devem ser realizados na menor altitude possível. Treinando em baixa altitude, as melhoras nos parâmetros fisiológicos são maximizados em relação ao treinamento feito exclusivamente em alta altitude. Enquanto viagens para grandes altitudes (2.000-2.500m) sem um deslocamento para realização de treinos intensos, ou o uso de simuladores de altitude por períodos menores que 12h, podem ser logisticamente mais fáceis, os diversos dados sugerem que esses métodos, na prática, podem ser inúteis em aumentar a oferta de oxigênio e melhorar o rendimento aeróbio. É importante também ressaltar que a resposta a determinada altitude pode variar de um individuo para o outro(36).

\section{Condições ambientais}

Competições esportivas de longa duração são realizadas em diferentes condições ambientais, que apresentam variação de temperatura, umidade, sol, vento, chuva. Tais condições atuam em conjunto com a intensidade do exercício e duração da prova, acentuando em algumas situações o ganho e em outras a perda de calor corporal. Se a temperatura corporal se eleva ou diminui fora dos limites desejáveis, tanto o rendimento quanto a saúde do atleta podem ser comprometidos ${ }^{(38)}$. Em ambientes quentes a temperatura da pele e a taxa de sudorese aumentam. Em tais situações, há maior fluxo sanguíneo para a pele a fim de favorecer a perda de calor, aumentando o esforço do sistema circulatório e da termorregulação ${ }^{(39,40)}$. Se uma parte do fluxo sanguíneo é direcionada à pele com o objetivo de aumentar a perda de calor, reduz-se o fluxo sanguíneo para a musculatura, acarretando diminuição do rendimento físico. Se o atleta insiste em manter o esforço, o fluxo sanguíneo que irriga a pele não conseguirá trocar todo o calor necessário, ocorrendo assim um acúmulo de calor no organismo. Além disso, o menor fluxo sanguíneo muscular poderá acarretar aumento na utilização do metabolismo anaeróbio e maior diminuição do glicogênio muscular ${ }^{(39)}$. Todos esses aspectos podem ser ainda exacerbados pela desidratação, que é comum durante competições no calor. A desidratação acarreta sobrecarga ainda maior ao sistema cardiovascular, diminuindo o volume de ejeção e aumentando a frequência cardíaca $(F C)^{(40,41,42)}$.
Duas estratégias têm se mostrado particularmente efetivas para reduzir os problemas de saúde e a diminuição do desempenho durante o exercício no calor: a aclimatação ao calor e a hidratação durante o exercício. As exposições regulares a ambientes quentes resultam em um número de adaptações fisiológicas que reduzem os efeitos negativos associados ao exercício no calor ${ }^{(43)}$. Essas adaptações incluem diminuição da temperatura interna do corpo no repouso, diminuição da FC durante o exercício, aumento da taxa de sudorese, menor temperatura interna para início da sudorese, melhora da distribuição do suor sobre a pele, diminuição da perda de sódio no suor e na urina, e expansão do volume plasmático ${ }^{(39,43)}$. Aclimatação ao calor seria completa apenas se a exposição for acompanhada pelo treinamento. Para que esta aconteça da melhor maneira, parecem necessárias elevação da temperatura interna do corpo e taxa de sudorese de no mínimo 400-600ml por hora em temperaturas maiores que $30^{\circ} \mathrm{C}^{(39)}$. Aclimatação é também específica à umidade do ar; exposições ao calor em ambientes secos proporcionam somente aclimatação parcial aos ambientes quentes e úmidos. A seguir estão listadas recomendações práticas para aclimatação ao calor:

- Uma completa adaptação demorará de sete a 14 dias.

- Melhor aclimatação ao calor é alcançada realizando treinamentos intervalados intensos ou exercícios contínuos acima de $50 \% \mathrm{VO}_{2}$ max por no mínimo uma hora a cada três dias. Exposição diária ao calor pode acelerar esse processo.

- Exercícios de aproximadamente 1,5-2h parecem ser mais efetivos para induzir aclimatação.

- As respostas à aclimatação são mantidas por no mínimo uma semana, mas elas podem durar até um mês.

Os aspectos relacionados com a hidratação durante o exercício podem ser aprofundados em Von Duvillard et al. ${ }^{(41)}$ e Murray et al. ${ }^{(40)}$.

\section{Aquecimento}

Aquecimento é uma prática muito comum, que precede todos os eventos atléticos. Enquanto o aquecimento é considerado ser essencial para o rendimento máximo por muitos técnicos e atletas, existem poucas evidências disponíveis suportando sua eficácia. O aquecimento ativo parece melhorar o rendimento aeróbio se ele permite que o atleta comece o esforço subsequente (competição) sem estar fadigado. Um aumento da temperatura muscular associado ao aquecimento pode melhorar o desempenho de muitas maneiras. Tem sido demonstrado que o aumento da temperatura diminui a rigidez dos músculos e articulações, aumenta a taxa de transmissão dos impulsos nervosos e a glicogenólise e a glicólise ${ }^{(44,45)}$. Além dos efeitos sobre a temperatura, o aquecimento ativo pode também aumentar o fluxo sanguíneo da musculatura e mantê-lo aumentado por alguns minutos após seu término(46). Contudo, apesar desses efeitos positivos, é importante que o aquecimento não seja muito intenso ou seguido de um inadequado período de recuperação.

Realmente, enquanto o aquecimento tem demonstrado melhorar o desempenho aeróbio, ele pode ter um efeito maléfico se causar depleção de substratos ou significante aumento na solicitação da termorregulação. $\mathrm{O}$ aquecimento causaria provavelmente aumento da temperatura corporal, fazendo com que o atleta inicie a competição com temperatura corporal mais elevada. Em condições de temperatura e umidade ambiente elevada, iniciar a atividade com a temperatura corporal mais próxima dos valores críticos pode prejudicar o rendimento e causar maior sobrecarga sobre o sistema termoregulatório e cardiovascular ${ }^{(38)}$ Já o esfriamento do corpo com imersão em água fria ou esfriamento do ar pode ser benéfico nessas condições onde o acúmulo de calor no organismo pode ser prejudicial para o rendimento ${ }^{(45)}$. No entanto, o acesso a esse tipo de prática não parece tão fácil de ser realizada em ambientes de competição e, em alguns casos, onde a competição se inicia com intensidades elevadas, poderia ser até prejudicial. 
Excluindo situações como descritas anteriormente, para melhorar o rendimento aeróbio é importante estruturar um aquecimento que seja de suficiente intensidade, duração e recuperação, sem causar fadiga significante. Enquanto um aquecimento ótimo depende de muitos fatores, parece que um aquecimento realizado a $60-80 \%$ do $\mathrm{VO}_{2}$ max por 5-10min, seguido por período de recuperação igual ou menor que 10min pode melhorar o desempenho aeróbio(45,46). Além disso, técnicas de aquecimento passivo (com cobertores térmicos) podem ser importantes para manter o aumento da temperatura produzida pelo aquecimento ativo, especialmente se existe um inevitável atraso entre o aquecimento e o início da competição ou se o tempo é frio(46).

\section{CONSIDERAÇÕES FINAIS}

O entendimento dos mecanismos de fadiga e das respostas fisiológicas associadas às diferentes durações e intensidades de exercício é essencial para uma correta elaboração das sessões de treinamento, mesmo quando esse treinamento é prescrito utilizando os diferentes índices de aptidão funcional. Através desse conhecimento, torna-se possível controlar variáveis importantes para o treinamento (frequência, volume, intensidade e recuperação) visando melhora contínua do rendimento aeróbio, sem prejudicar a saúde do atleta. Além disso, cuidados no planejamento do aquecimento antes de uma competição e de correta aclimatação ao calor durante os treinamentos para competições em ambientes quentes e úmidos podem ser importantes ferramentas para otimizar o rendimento aeróbio.

Para atingir um ótimo rendimento atlético, seria essencial chegar o mais próximo possível do limite tolerável da carga de treinamento, adquirindo assim as maiores adaptações possíveis. Obviamente, ter o conhecimento exato desse limite não é fácil ou talvez impossível; no entanto, algumas sugestões podem ajudar na tentativa de otimizar o treinamento e rendimento aeróbio: 1)Treinos intervalados de alta intensidade (uma ou duas vezes por semana) são imprescindíveis para melhora de rendimento em atletas altamente treinados; 2) Manter um histórico das cargas de treino e recuperações; 3) Aprender com a experiência os tipos de cargas que podem ser toleradas individualmente; essa é uma das vantagens de trabalhar com atletas durante anos; 4) Não esperar até que a fadiga seja excessiva, mas planejar cuidadosamente períodos frequentes de recuperação; 5) Realizar a próxima sessão de treinamento intenso somente após um adequado período de recuperação.

Todos os autores declararam não haver qualquer potencial conflito de interesses referente a este artigo.

\section{REFERÊNCIAS BIBLIOGRÁFICAS}

1. Jones AM, Koppo K, Burnley M. Effects of prior exercise on metabolic and gas exchange responses to exercise. Sports Med 2003;33:949-71

2. Londeree BR. Effect of training on lactate/ventilatory thresholds: a meta-analysis. Med Sci Sports Exerc 1997;29:837-43

3. Gibala MJ, Little JP, van Essen M, Wilkin GP, Burgomaster KA, Safdar A, et al. Short-term sprint interval versus traditional endurance training: similar initial adaptations in human skeletal muscle and exercise performance. J Physiol 2006;575:901-11.

4. Lindsay FH, Hawley JA, Myburgh KH, Schomer HH, Noakes TD, Dennis SC. Improved athletic performance in highly trained cyclists after interval training. Med Sci Sports Exerc 1996;28:1427-34

5. Billat LV. Interval training for performance: a scientific and empirical practice. Special recommendations for middle-and long-distance running. Part I: aerobic interval training. Sports Med 2001;31:13-31.

6. Billat LV. Use of blood lactate measurements for prediction of exercise performance and for control of training. Recommendations for long-distance running. Sports Med 1996;22:157-75.

7. Poole DC, Gaesser GA. Response of ventilatory and lactate thresholds to continuous and interval training. J Appl Physiol 1985;58:1115-21.

8. Denadai BS, Ortiz MJ, Greco CC, de Mello MT. Interval training at $95 \%$ and $100 \%$ of the velocity at $\mathrm{VO}_{2}$ max: effects on aerobic physiological indexes and running performance. Appl Physiol Nutr Metab 2006;31:737-43.

9. Laursen $P B$, Jenkins DG. The scientific basis for high-intensity interval training: optimising training programmes and maximising performance in highly trained endurance athletes. Sports Med 2002;32:53-73.

10. Laursen PB, Shing CM, Peake JM, Coombes JS, Jenkins DG. Influence of high-intensity interval training on adaptations in well-trained cyclists. J Strength Cond Res 2005;19:527-33.

11. Pascoe DD, Gladden LB. Muscle glycogen resynthesis after short term, high intensity exercise and resistance exercise. Sports Med 1996;21:98-118.

12. Bickham DC, Bentley DJ, Le Rossignol PF, Cameron-Smith D. The effects of short-term sprint training on MCT expression in moderately endurance-trained runners. Eur J Appl Physiol 2006;96:636-43.

13. Astrand PO, Rodahl K. Textbook of work physiology. New York: McGraw Hill; 1977.

14. Hargreaves M, McKenna MJ, Jenkins DG, Warmington SA, Li JL, Snow RJ, et al. Muscle metabolites and performance during high-intensity, intermittent exercise. J Appl Physiol 1998;84:1687-91.

15. di Prampero PE. Energetics of muscular exercise. Rev Physiol Biochem Pharmacol 1981;89:143-222

16. Gastin PB. Energy system interaction and relative contribution during maximal exercise. Sports Med 2001;31:725-41. Review.

17. Layzer RB. Muscle metabolism during fatigue and work. Baillieres Clin Endocrinol Metab 1990;4:441-59

18. Glaister M. Multiple sprint work: physiological responses, mechanisms of fatigue and the influence of aerobic fitness. Sports Med 2005;35:757-77.

19. Jentjens $R$, Jeukendrup A. Determinants of post-exercise glycogen synthesis during short-term recovery. Sports Med 2003;33:117-44

20. Gibala MJ, McGee SL. Metabolic adaptations to short-term high-intensity interval training: a little pain for a lot of gain? Exerc Sport Sci Rev 2008;36:58-63. Review.

21. Messonnier $L$, Freund $H$, Denis $C$, Feasson L, Lacour JR. Effects of training on lactate kinetics parameters and their influence on short high-intensity exercise performance. Int J Sports Med 2006;27:60-6.

22. Counsilman JE. The science of swimming. London: Pelham Books, 1968

23. da Nobrega AC. The subacute effects of exercise: concept, characteristics, and clinical implications Exerc Sport Sci Rev 2005;33:84-7.
24. Billat $\mathrm{V} L$, Flechet $\mathrm{B}$, Petit $\mathrm{B}$, Muriaux $\mathrm{G}$, Koralsztein JP. Interval training at $\mathrm{VO}_{2}$ max: effects on aerobic performance and overtraining markers. Med Sci Sports Exerc 1999;31:156-63.

25. Fry AC, Kraemer WJ. Resistance exercise overtraining and overreaching. Neuroendocrine responses. Sports Med 1997;23:106-29. Review.

26. Hansen AK, Fischer CP, Plomgaard P, Andersen JL, Saltin B, Pedersen BK. Skeletal muscle adaptation: training twice every second day vs. training once daily. J Appl Physiol 2005;98:93-9.

27. Yeo WK, Paton CD, Garnham AP, Burke LM, Carey AL, Hawley JA. Skeletal muscle adaptation and performance responses to once a day versus twice every second day endurance training regimens. J Appl Physiol 2008;105:1462-70.

28. Tee JC, Bosch AN, Lambert MI. Metabolic consequences of exercise-induced muscle damage. Sports Med 2007;37:827-36.

29. Nieman DC. Marathon training and immune function. Sports Med 2007;37:412-5.

30. Asp S, Rohde T, Richter EA. Impaired muscle glycogen resynthesis after a marathon is not caused by decreased muscle GLUT-4 content. J Appl Physiol 1997;83:1482-5.

31. Warhol MJ, Siegel AJ, Evans WJ, Silverman LM. Skeletal muscle injury and repair in marathon runners after competition. Am J Pathol 1985;118:331-80.

32. Hinds T, McEwan I, Perkes J, Dawson E, Ball D, George K. Effects of massage on limb and skin blood flow after quadriceps exercise. Med Sci Sports Exerc 2004;36:1308-13.

33. Midgley AW, McNaughton LR, Jones AM. Training to enhance the physiological determinants of longdistance running performance: can valid recommendations be given to runners and coaches based on current scientific knowledge? Sports Med 2007;37:857-80

34. Faria EW, Parker DL, Faria IE. The science of cycling: physiology and training - part 1. Sports Med 2005;35:285-312.

35. Chapman RF, Stray-Gundersen J, Levine BD. Individual variation in response to altitude training. J Appl Physiol 1998;85:1448-56.

36. Levine BD, Stray-Gundersen J. "Living high-training low": effect of moderate-altitude acclimatization with low-altitude training on performance. J Appl Physiol 1997;83:102-12.

37. Chapman R, Levine BD. Altitude training for the marathon. Sports Med 2007;37:392-5.

38. Tucker R. Thermoregulation, fatigue and exercise modality. Med Sport Sci 2008;53:26-38.

39. Wendt $\mathrm{D}$, van Loon $L$, Lichtenbelt WD. Thermoregulation during exercise in the heat: strategies for maintaining health and performance. Sports Med 2007;37:669-82.

40. Murray B. Hydration and physical performance. J Am Coll Nutr 2007;26:542-8. Review.

41. Von Duvillard SP, Braun WA, Markofski M, Beneke R, Leithauser R. Fluids and hydration in prolonged endurance performance. Nutrition 2004;20:651-6.

42. Maughan RJ, Shirreffs SM. Development of individual hydration strategies for athletes. Int I Sport Nutr Exerc Metab 2008;18:457-72. Review.

43. Sunderland C, Morris JG, Nevill ME. A heat acclimation protocol for team sports. Br J Sports Med 2008:42:327-33.

44. Febbraio MA, Carey MF, Snow RJ, Stathis CG, Hargreaves M. Influence of elevated muscle temperature on metabolism during intense, dynamic exercise. Am J Physiol 1996;271:1251-5.

45. Uckert S, Joch W. Effects of warm-up and precooling on endurance performance in the heat. $\mathrm{Br} J$ Sports Med 2007;41:380-4.

46. Bishop D. Warm up II: performance changes following active warm up and how to structure the warm up. Sports Med 2003:33:483-98. 